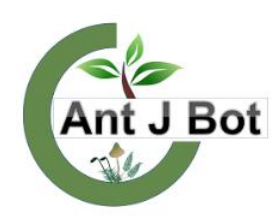

Received : 16.01.2018 Accepted : 03.02.2018

\section{New bryophillic Pyronemataceae records for Turkish Pezizales from Gaziantep province}

\author{
Yasin UZUN ${ }^{1 *}$, İbrahim Halil KARACAN ${ }^{2}$, Semiha YAKAR ${ }^{1}$, Abdullah KAYA ${ }^{1}$ \\ ${ }^{1}$ Karamanoğlu Mehmetbey University, Kâmil Özdă̆ Science Faculty, Department of Biology, Karaman, Turkey \\ ${ }^{2}$ Ömer Özmimar Religious Anatolian High School, Gaziantep, Turkey \\ *yasinuzun_61@hotmail.com
}
Türkiye Pezizales'leri için Gaziantep'ten yeni briyofilik Pyronemataceae kayitları

\begin{abstract}
This study was based on fourteen bryophilous Pyronemataceae species. Thirteen of them (Inermisia gyalectoides (Svrček \& Kubička) Dennis \& Itzerott, Lamprospora carbonicola Boud., Lamprospora dictydiola Boud., Lamprospora miniata De Not., Octospora areolata (Seaver) Caillet \& Moyne, Octospora axillaris (Nees) M.M. Moser, Octospora coccinea (P. Crouan \& H. Crouan) Brumm., Octospora excipulata (Clem.) Benkert, Octospora gemmicola Benkert, Octospora muscimuralis Graddon, Octospora orthotrichi (Cooke \& Ellis) K.B. Khare \& V.P. Tewari, Octospora polytrichi (Schumach.) Caillet \& Moyne and Octospora rustica (Velen.) J.Moravec) are given as new records for the macromycota of Turkey. Inermisia and Lamprospora are new at genus level. New localities are given for the $14^{\text {th }}$ species, Octospora leucoloma Hedw. Brief descriptions about the macroscopic and microscopic characters of the species and their photographs are provided.
\end{abstract}

Key words: Biodiversity, bryoparasitic fungi, Pyronemataceae, new records, Turkey

Özet: Bu çalışma 14 briyofilik Pyronemataceae türü üzerinde temellendirilmiştir. Bunlardan 13 tanesi (Inermisia gyalectoides (Svrček \& Kubička) Dennis \& Itzerott, Lamprospora carbonicola Boud., Lamprospora dictydiola Boud., Lamprospora miniata De Not., Octospora areolata (Seaver) Caillet \& Moyne, Octospora axillaris (Nees) M.M. Moser, Octospora coccinea (P. Crouan \& H. Crouan) Brumm., Octospora excipulata (Clem.) Benkert, Octospora gemmicola Benkert, Octospora muscimuralis Graddon, Octospora orthotrichi (Cooke \& Ellis) K.B. Khare \& V.P. Tewari, Octospora polytrichi (Schumach.) Caillet \& Moyne ve Octospora rustica (Velen.) J.Moravec) Türkiye makromikotası için yeni kayıttır. Inermisia ve Lamprospora cins düzeyinde yenidir. On dördüncü türe (Octospora leucoloma Hedw.) ilişkin yeni lokaliteler verilmiştir. Türlerin makroskobik ve mikroskobik karakterlerine ilişkin kısa betimlemeleri ve fotoğrafları verilmiştir.

Anahtar kelimeler: Biyoçeşitlilik, bryoparazitik mantarlar, Pyronemataceae, yeni kayıtlar, Türkiye

\section{Introduction}

Fungi are heterotrophic organisms growing on either dead organic material or on different organs of some other living organisms. Those growing on gametophytic or sporophytic organs of bryophytes are usually known as bryophilous fungi. Within the order Pezizales the members of the genera Filicupula Y.J.Yao \& Spooner, Hiemsia Svrček, Inermisia Rifai, Lamprospora De Not, Neottiella (Cooke) Sacc., Octospora Hedw., Octosporella Döbbeler, Octosporopsis U.Lindem. \& M.Vega and Ramsbottomia W.D.Buckley are known to have bryophilous mode of life (Felix, 1988; Benkert, 2007; Kirk et al., 2008). Except the genus Filicupula all of them are currently positioned in Pyronemataceae.

Since they require similar environmental conditions, moss and fungus could also live together. Bryophytes are capable of supporting fungal growth by providing a good microenvironment (Stephenson \& Studlar 1985). Some fungi within the order Pezizales, especially the members of the genera Octospora, prefer small, acrocarpous mosses as substrates that can live in unfavorable conditions like a rocky or hot and frequently dry surfaces (Hughes, 1982). Though research on bryoparasitic species has a long tradition in some European countries (Egertova et al., 2015) and the presence of about 300 species of ascomycetes growing on the gametophytes of mosses or hepatics were reported (Döbbeler, 1997), bryophilous fungi has received almost no attention in Turkey. According to the current checklists (Sesli and Denchev,
2014; Solak et al., 2015) 31 basidiomycete species growing on or around the bryophytes are known to exist in Turkey. But only six bryophilous ascomycete species, Marcelleina persoonii (P.Crouan \& H.Crouan) Brumm. (Pezizaceae) (Y1lmaz et al., 1997), Neottiella rutilans (Fr.) (Akata and Kaya, 2013), Octospora itzerottii Benkert (Uzun et al., 2017), O. leucoloma Hedw. (Çolak and Kaygusuz, 2017), Scutellinia trechispora (Berk. \& Broome) Lambotte (Pyronemataceae) (Kaya et al., 2016) and Pseudoplectania sphagnophila (Pers.) Kreisel (Sarcosomataceae) (Türkoğlu and Yağız, 2012), have so far been recorded from Turkey yet. Compared to 81 octosporoid fungi existing in Europe (Benkert, 2007), there is still much to be done in Turkey.

The work aims to make a contribution to the mycobiota of Turkey by adding new bryophilous ascomycete species.

\section{Materials and methods}

Macrofungi samples were collected from the different localities within the boundaries of Gaziantep province between 2013 and 2017. Geographic positions were noted together with their necessary morphological and ecological characteristics, and they were photographed in their natural habitats. Olympus SZX7 trinocular stereomicroscope was used for some macrostructural properties of smaller fruit bodies. Nikon eclipse Ci-S trinocular light microscope, DS-Fi2 digital camera and Nikon DS-L3 displaying apparatus were used for microstructural investigation and photographing. Identification of the samples were performed with the help 
of Seaver (1912, 1914, 1942), Le Gal (1940), Svrček and Kubička (1961), Dougoud and Roth (1972), Dennis and Itzerott (1973), Khare and Tewari (1975), Khare (1976, 2003), Breitenbach and Kränzlin (1984), Senn-Irlet (1988), Wang and Kimbrough (1992), Schumacher (1993), Fox et al. (1994), Benkert (1987, 1997, 1998), Yao and Spooner (1996), Hansen and Pfister (2006), Medardi (2006), Eckstein and Eckstein (2009, 2013), Eckstein (2014), Pradhan et al. (2013), Wieschollek (2013), Beug et. al. (2014), Eckstein et al., (2014) and Egertova et al., (2015).

\section{Results}

The systematics of the taxa are given in accordance with Cannon and Kirk (2007), Kirk et al. (2008), and the Index Fungorum (www.indexfungorum.org; accessed 02 November 2017). The taxa are listed in alphabetical order with brief descriptions, habitats, localities, collection dates and accession numbers.

Ascomycota Whittaker

Pezizales J. Schröt.

\section{Pyronemataceae Corda}

3.1. Inermisia gyalectoides (Svrček \& Kubička) Dennis \& Itzerott, Kew Bull. 28(1): 22 (1973) (Figure 1)

Syn. Octospora gyalectoides Svrček \& Kubička.

Macroscopic and microscopic features: Apothecia 1-2 $\mathrm{mm}$ in diameter, more or less convex, pale orange to orange, sometimes with lighter margin. Asci 180-240 $\times$ 15-18 $\mu \mathrm{m}$, cylindrical, eight spored, spores uniseriate. Paraphyses cylindrical, enlarged towards the apex, some forked above. Spores 16.5-22 × 9-12.5 $\mu \mathrm{m}$, ellipsoid, smooth, hyaline, with a large oil drop. Inermisia gyalectoides grows in association with scattered small mosses, Bryum argenteum Hedw., Funaria hygrometrica Hedw, Pterygoneurum ovatum (Hedw.) Dixon and Pottia (Reichenbach) Fürnrohr sp. (Dennis and Itzerott, 1973; Benkert, 2007; Eckstein and Eckstein, 2009).

Specimen examined: Gaziantep, Şehitkâmil, city cemetery, on Pterygoneurum ovatum, $37^{\circ} 04^{\prime} \mathrm{N}, 37^{\circ} 23^{\prime} \mathrm{E}$, $845 \mathrm{~m}, \quad 04.01 .2015$, K.11164; Öğümsöğüt village, $37^{\circ} 06^{\prime} \mathrm{N}, 37^{\prime} 18^{\circ} \mathrm{E}, 1020 \mathrm{~m}, 07.03 .2015, \mathrm{~K} .11409$.

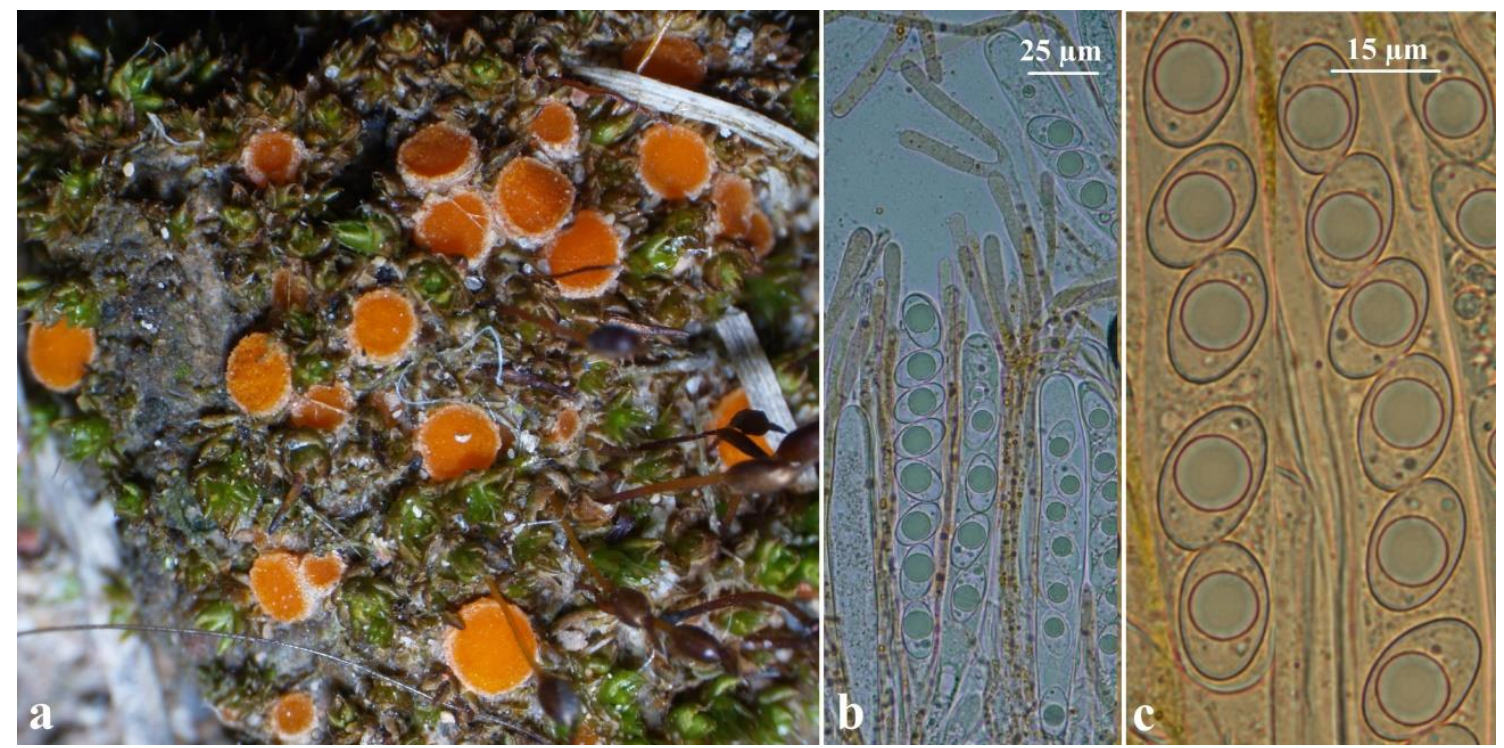

Figure 1. Inermisia gyalectoides: a- ascocarps, b- asci and paraphyses, c- ascospores.

3.2. Lamprospora carbonicola Boud., Hist. Class. Discom. Eur. (Paris): 68 (1907) (Figure 2)

Syn. Barlaeina carbonicola (Boud.) Sacc. \& Traverso; Octospora carbonicola (Boud.) Yei Z. Wang.

Macroscopic and microscopic features: Apothecia 1-4 $\mathrm{mm}$ in diameter, broad, partly submerged in the soil among shoots of its host, hymenium orange to bright red, with conspicious membranaceous margin. Asci 180-260 $\times$ 16-19 $\mu \mathrm{m}$, cylindrical, tapering towards the base. Paraphyses straight, septate, enlarged at the tip with orange, granular content. Spores 13-16 $\mu \mathrm{m}$, globose to subglobose, with a ridged ornamentation forming a finemeshed reticulum, and a large oil drop. Lamprospora carbonicola grows among the moss Funaria Hedwig, Tortula Hedw. (Wang and Kimbrough, 1992), Pholia Hedw., Barbula Hedw. sp. (Schumacher, 1993) on moist, burnt sandy gravelly forest floor in shade of trees (Pradhan et al., 2013).
Specimen examined: Gaziantep, Nizip, Sekili village, on cemetery wall with Funaria hygrometrica Hedw., $36^{\circ} 58^{\prime} \mathrm{N}, \quad 37^{\circ} 40^{\prime} \mathrm{E}, \quad 600 \mathrm{~m}, \quad 28.02 .2015, \quad$ K.11340; Şehitkâmil, central city cemetery, $37^{\circ} 04^{\prime} \mathrm{N}, 37^{\circ} 23^{\prime} \mathrm{E}, 845$ $\mathrm{m}, 06.03 .2015$, K.11383; Oğuzeli, cemetery, $36^{\circ} 57^{\prime} \mathrm{N}$, $37^{\circ} 30^{\prime} \mathrm{E}, 670 \mathrm{~m}, 08.03 .2015$, K.11427; Nurdağı, Atalar village, $37^{\circ} 08^{\prime} \mathrm{N}, 36^{\circ} 54^{\prime} \mathrm{E}, 985 \mathrm{~m}, 22.03 .2015$, K. 11497.

3.3. Lamprospora dictydiola Boud., Hist. Class. Discom. Eur. (Paris): 68 (1907) (Figure 3)

Syn. Barlaeina dictydiola (Boud.) Sacc. \& Traverso; Octospora dictydiola (Boud.) Caillet \& Moyne.

Macroscopic and microscopic features: Apothecia 1-2 $\mathrm{mm}$ in diameter, broad, shallow cupulate to turbinate, partly submerged among shoots of its host, hymenium orange to orange red, with prominent membranaceous margin. Asci 170-200 × 17-19 $\mu \mathrm{m}$, cylindrical, gradually tapering towards the base. Paraphyses straight, sparsely branched from below, enlarged at the tip with orange, granular content. Spores 14-16 × 13.5-15.5 $\mu \mathrm{m}$, globose to 
subglobose, with a ridged ornamentation forming a finemeshed reticulum, and a large oil drop. Lamprospora dictydiola was reported to grow on vertical parts of old garden wall among the moss Tortula muralis L. ex Hedw. (Benkert and Brouwer, 2004).
Specimen examined: Gaziantep, Şehitkâmil, central city cemetery, on Tortula muralis, $37^{\circ} 04^{\prime} \mathrm{N}, 37^{\circ} 23^{\prime} \mathrm{E}, 860 \mathrm{~m}$, 27.03.2015, K.11503; Islahiye, Tandır village, Huzurlu high plateau, near the road, $36^{\circ} 58^{\prime} \mathrm{N}, 36^{\circ} 30^{\prime} \mathrm{E}, 1410 \mathrm{~m}$, K.11899.
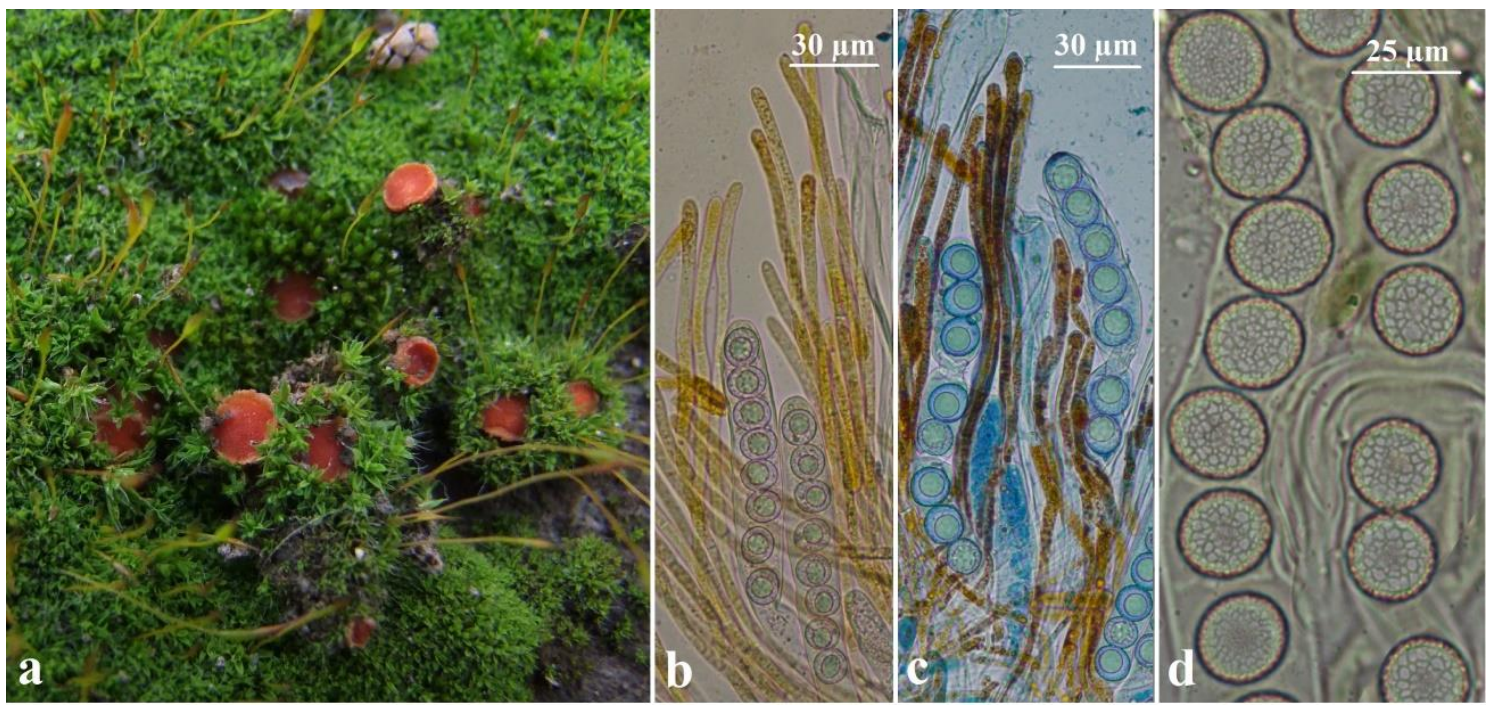

Figure 2. Lamprospora carbonicola: a- ascocarps, b,c- asci and paraphyses, d- ascospores.
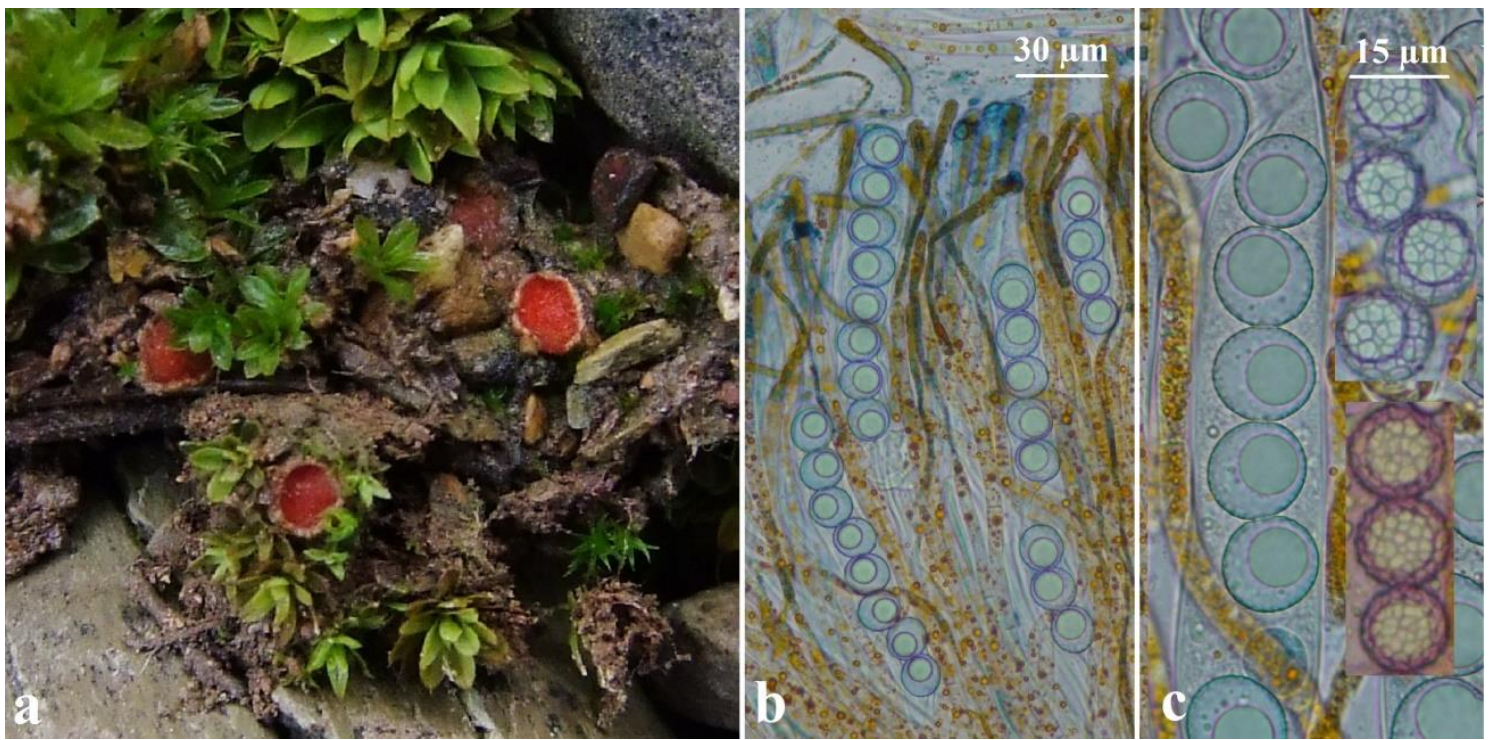

Figure 3. Lamprospora dictydiola: a- ascocarps, b- asci and paraphyses, c- ascospores.

3.4. Lamprospora miniata De Not., Comm. Soc. crittog. Ital. 1(fasc. 5): 388 (1864) (Figure 4)

Syn. Ascobolus miniatus P. Crouan \& H. Crouan; Barlaea crouanii (Cooke) Massee; Barlaea miniata (De Not.) Sacc.; Barlaeina miniata (De Not.) Sacc. \& Traverso; Crouania crouanii (Cooke) Lambotte; Crouania miniata (De Not.) Fuckel; Humaria crouanii (Cooke) Quél.; Lamprospora crouanii (Cooke) Seaver, Lamprospora crouanii (Cooke) Seaver f. crouanii; Lamprospora crouanii f. magnihyphosa J. Moravec; Lamprospora miniata De Not.; Lamprospora miniata De Not. f. miniata; Lamprospora miniata f. parvispora Benkert; Lamprospora miniata De Not. var. miniata; Lamprospora miniata var. parvispora Benkert; Lamprospora miniata var. ratisbonensis Benkert; Octospora miniata (De Not.) Caillet \& Moyne; Peziza crouanii Cooke.
Macroscopic and microscopic features: Apothecia 1-5 $\mathrm{mm}$ in diameter, subglobose at first, expanding and becoming concave to almost plane with a slightly elevated margin when mature. Bright red, margin lighter. Asci 180$260 \times 18-22 \mu \mathrm{m}$, cylindrical, eight spored, spores uniseriate. Paraphyses cylindrical, somewhat thickened above. Spores 15-17 $\mu \mathrm{m}$ in diameter, globose, hyaline, with a large oil drop and ridged ornamentation when mature. Lamprospora miniata grows in association with Pottia bryoides (Dicks.) Mitt. and Phascum cuspidatum Schreb. ex Hedw. (Eckstein and Eckstein, 2009).

Specimen examined: Gaziantep, Nizip, Sekili village, cemetery, on soil with Phascum cuspidatum, $36^{\circ} 58^{\circ} \mathrm{N}$, $37^{\circ} 40^{\prime} \mathrm{E}, 600 \mathrm{~m}, 14.12 .2014, \mathrm{~K} .11110$; Oğuzeli, cemetery, $36^{\circ} 57^{\prime} \mathrm{N}, 37^{\circ} 30^{\prime} \mathrm{E}, 670 \mathrm{~m}, 05.04 .2015$, K.11664. 
3.5. Octospora areolata (Seaver) Caillet \& Moyne, Bull. trimest. Soc. mycol. Fr. 96(2): 199 (1980) (Figure 5)

Syn. Lamprospora areolata Seaver; Lamprospora areolata Seaver var. areolata; Octospora areolata (Seaver) Yei Z. Wang.

Macroscopic and microscopic features: Apothecia 0.5-1 $\mathrm{mm}$ in diameter, discoid to cupulate, hymenium orange red, light yellow when dry, margin slightly raised, outer surface darker and closely attached with moss. Asci 190-
$240 \times 18-21 \mu \mathrm{m}$, clavate-cylindrical, eight spored, spores uniseriate. Paraphyses subclavate, septate, slightly enlarged at apex up to 6-7 $\mu \mathrm{m}$. Spores 14-16 $\mu \mathrm{m}$ exluding ornamentation, globose, hyaline, marked with high ridges of 3-4 $\times 0.8 \mu \mathrm{m}$. Octospora areolata grows on soil among mosses (Wang and Kimbrough, 1992).

Specimen examined: Gaziantep, Nurdağ on Syntrichia ruralis (Hedw.) F.Weber \& D.Mohr, $37^{\circ} 10^{\prime} \mathrm{N}-36^{\circ} 40^{\prime} \mathrm{E}, 950 \mathrm{~m}, 10.04 .2015, \mathrm{~K} .11684$.
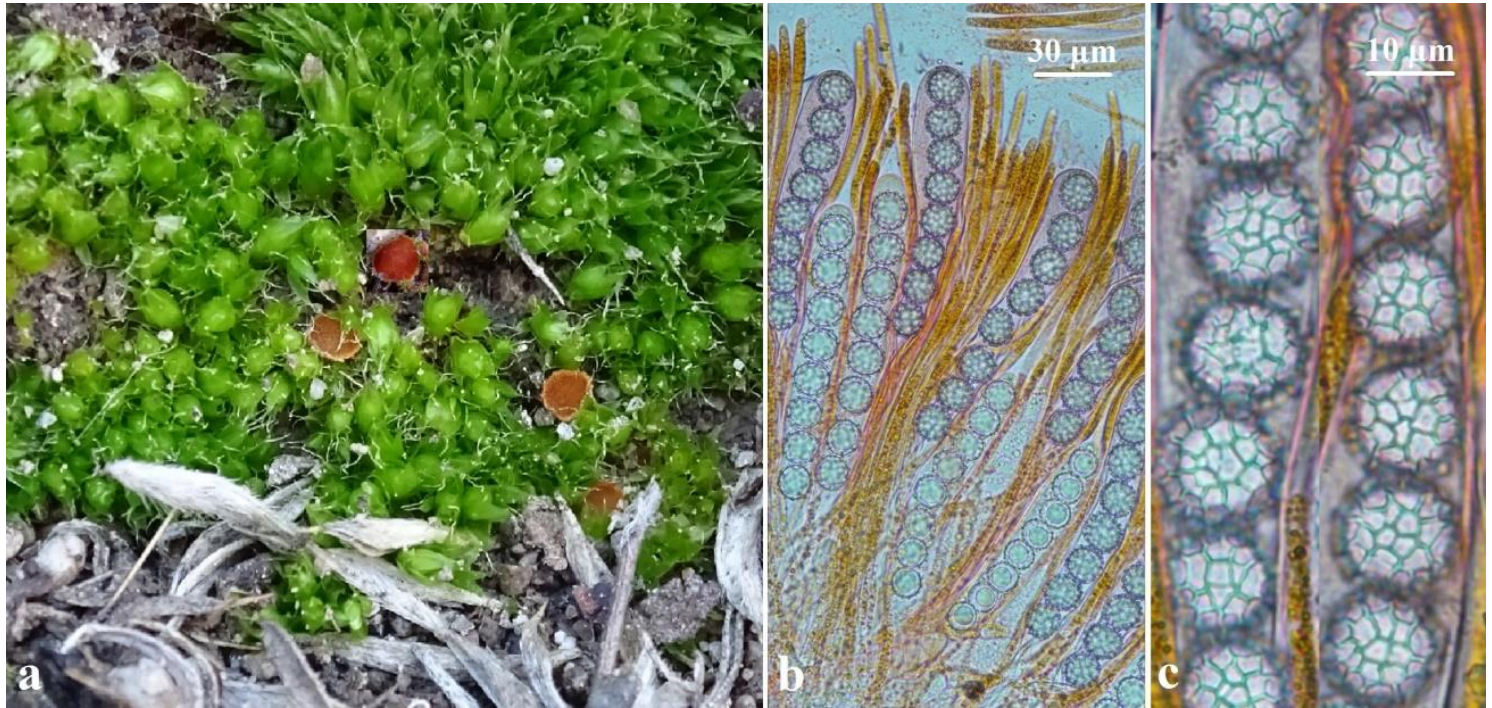

Figure 4. Lamprospora miniata: a- ascocarps, b- asci and paraphyses, c- ascospores.
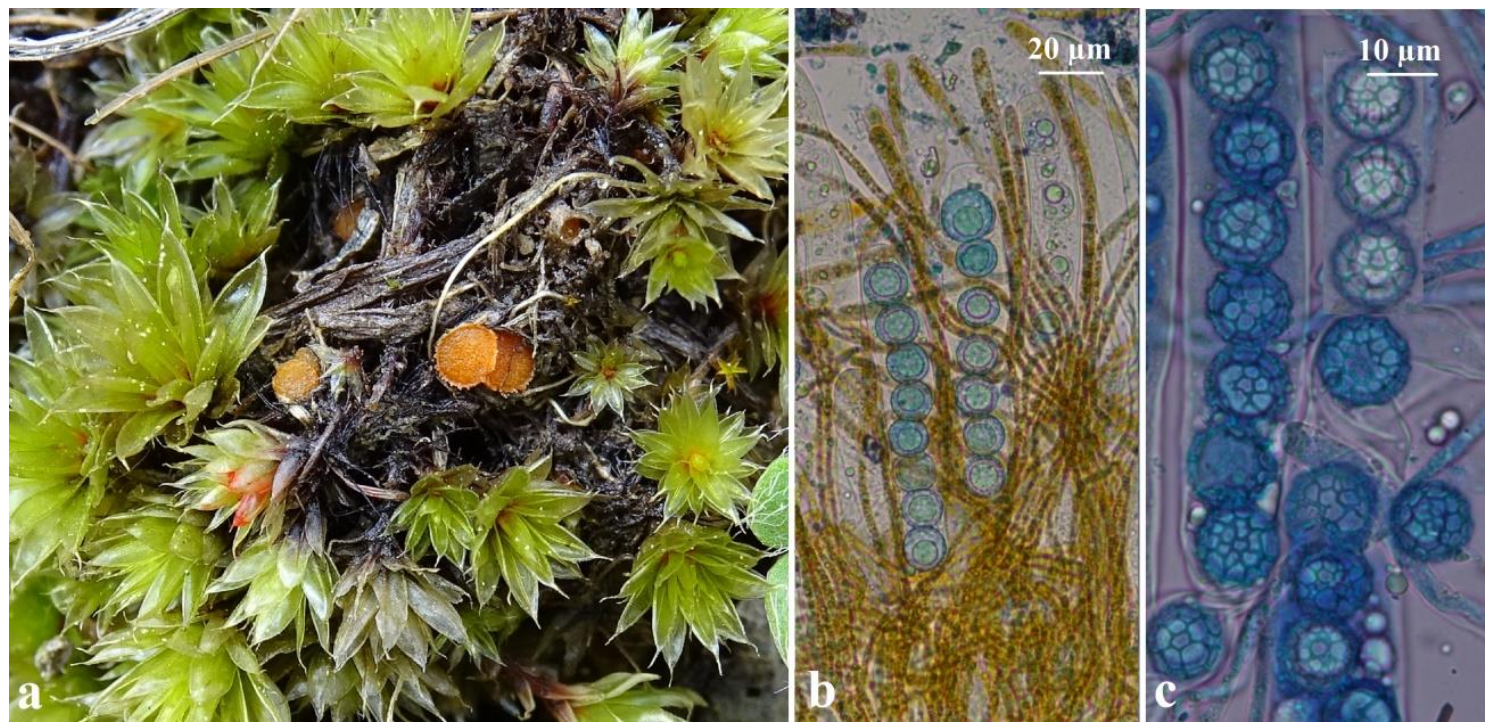

Figure 5. Octospora areolata: a- ascocarps, b- asci and paraphyses, c- ascospores.

3.6. Octospora axillaris (Nees) M.M. Moser, in Gams, Kl. Krypt.-Fl., Edn 3 (Stuttgart) 2a: 110 (1963) (Figure 6)

Syn. Helotium axillaris (Nees) Boud.; Humaria axillaris (Nees) Sacc.; Humaria carneola (Saut.) Sacc.; Humaria sublutea Velen.; Humarina axillaris (Nees) Seaver; Leucoloma axillaris (Nees) Fuckel; Octospora axillaris (Nees) M.M. Moser var. axillaris; Octospora axillaris var. dennisii Itzerott; Octospora axillaris var. tetraspora Benkert; Octospora carneola (Saut.) Dennis; Octospora sublutea (Velen.) Svrček; Peziza axillaris Nees; Peziza carneola Saut.
Macroscopic and microscopic features: Apothecia 0.5-2 (2.5) $\mathrm{mm}$ in diameter, flat to convex, sessile, orange. Asci $140-190 \times 18-22 \mu \mathrm{m}$, cylindrical, eight spored, somehow tapered toward the base, spores usually uniseriate. Paraphyses filiform, enlarged toward the apex up to 7-8 $\mu \mathrm{m}$. Spores 21-28 × 10-12 $\mu \mathrm{m}$, ellipsoid, hyaline, smooth, usually with two large oil drops. Octospora axillaris was reported to grow on soil among Barbula unguiculata Hedw., Entosthodon fascicularis (Hedw.) Müll. Hal., Pottia davalliana (Sm.) C.E.O.Jensen, Pottia lanceolata (Hedw.) Müll.Hal. (Caillet and Moyne, 1989), Phascum cuspidatum Schreb. ex Hedw., Syntrichia ruralis (Hedw.) F.Weber \& D.Mohr, Tortula ruralis (Hedw.) Gaertn. 
(Dennis and Itzerott, 1973; Benkert, 2007; Eckstein and Eckstein, 2009), and on the protonema of Dicranella heteromalla (Hedw.) Schimp. (Itzerott, 1977).
Specimen examined: Gaziantep, Islahiye, Hanağz1 village, on soil among Ptychostomum donianum (Grev.) Holyoak \& N.Pedersen, $37^{\circ} 03^{\prime} \mathrm{N}-36^{\circ} 36^{\prime} \mathrm{E}, 625 \mathrm{~m}$, 08.11.2014, K.10515; K.10517.
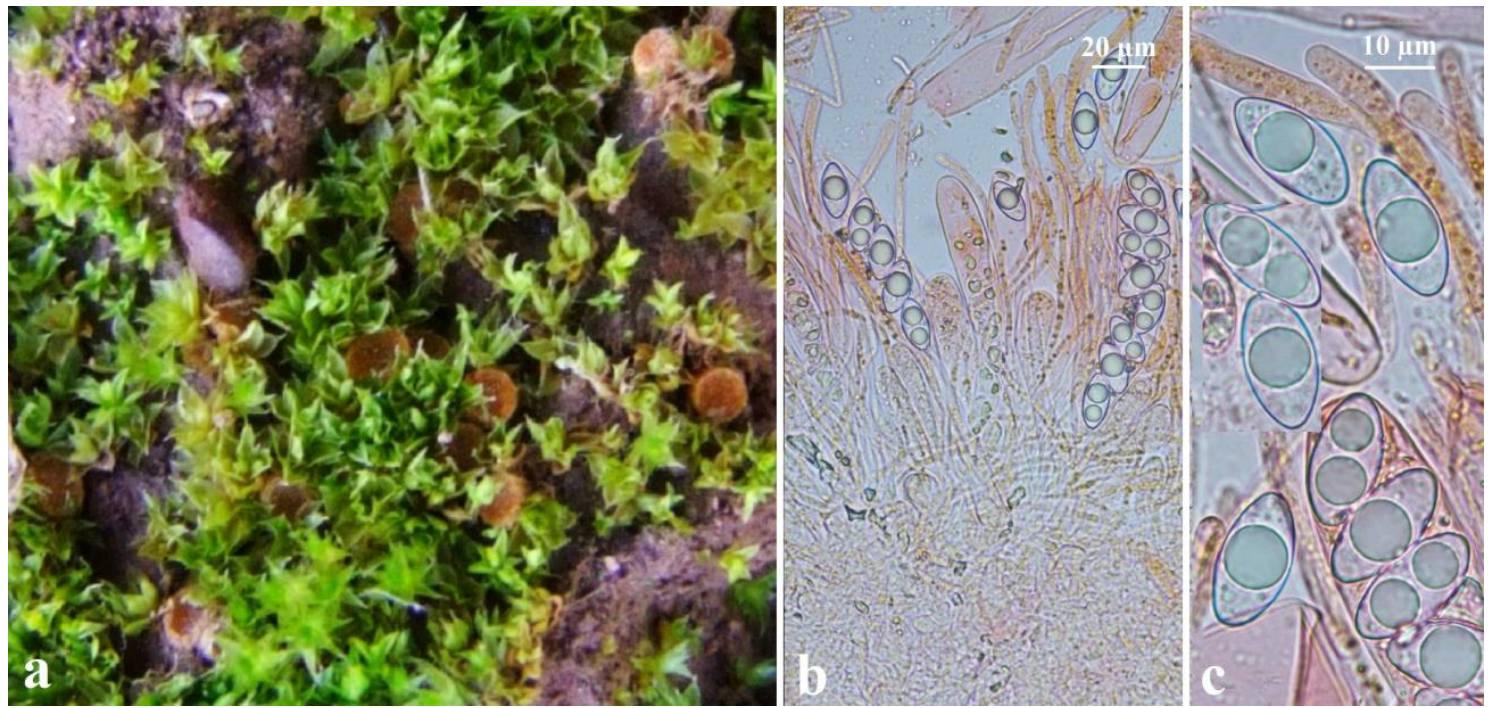

Figure 6. Octospora axillaris: a- ascocarps, b- asci and paraphyses, c- ascospores.

3.7. Octospora coccinea (P. Crouan \& H. Crouan) Brumm., Persoonia, Suppl. 1: 213 (1967) (Figure 7)

Syn. Ascobolus coccineus P. Crouan \& H. Crouan; Byssonectria coccinea (P. Crouan \& H. Crouan) M. Torre; Humaria coccinea (P. Crouan \& H. Crouan) Sacc.; Humaria coccinea var. maritima Grélet; Humaria muralis Quél.; Humarina coccinea (P. Crouan \& H. Crouan) Seaver; Humarina coccinea (P. Crouan \& H. Crouan) Seaver var. coccinea; Humarina coccinea var. maritima (Grélet) Cash; Neottiella corallina (Cooke) Massee; Octospora coccinea (P. Crouan \& H. Crouan) Brumm. var. coccinea; Octospora coccinea var. maritima (Grélet) Parrett. \& Gaggian.; Octospora coccinea var. tetraspora Benkert; Peziza corallina Cooke; Peziza muralis Quél.

Macroscopic and microscopic features: Apothecia 1-2 $\mathrm{mm}$ in diameter, cup to flat disk shaped, margin sometimes pubescent, hymenium orange. Asci 120-180 × 16-22 $\mu \mathrm{m}$, cylindrical to clavate, eight spored, spores biseriate. Paraphyses cylindrical, very slim, enlarged towards the apex up to 3-7 $\mu \mathrm{m}$, often slightly curved. Spores $25-27 \times 7.5-9.5 \mu \mathrm{m}$, narrowly ellipsoid to narrowly fusiform, smooth, hyaline, with two big drops in the middle and often small drops at the poles. Octospora coccinea grows mainly among Mniobryum sp. but also with Bryum argenteum Hedw., Bryum klinggraeffii Schimp., Ceratodon purpureus (Hedw.) Brid., Pottia sp. (Dennis and Itzerott, 1973; Eckstein and Eckstein, 2009), Encalypta vulgaris Hedw. (Benkert, 2007), Barbula unguiculata Hedw. and Oxyrrhynchium praelongum (Hedw.) Warnst. (Caillet and Moyne, 1989).

Specimen examined: Gaziantep, Şehitkamil, Cerityeniyapan village, among Bryum sp., $37^{\circ} 10^{\prime} \mathrm{N}-$ $37^{\circ} 08^{\prime} \mathrm{E}, 1070 \mathrm{~m}, 07.03 .2015$, K.11398; K.11402; Yavuzeli, Çimenli village, $37^{\circ} 16^{\prime} \mathrm{N}-37^{\circ} 34^{\prime} \mathrm{E}, 710 \mathrm{~m}$, 29.03.2015, K.11548; Nurdağ1, Kömürler village, roadside, $37^{\circ} 09^{\prime} \mathrm{N}-36^{\circ} 48^{\prime} \mathrm{E}, 535 \mathrm{~m}, 03.04 .2015$, K.11583; Atmalı village, $37^{\circ} 07^{\prime} \mathrm{N}-36^{\circ} 53^{\prime} \mathrm{E}, 700 \mathrm{~m}, 04.04 .2015$, K.11618.
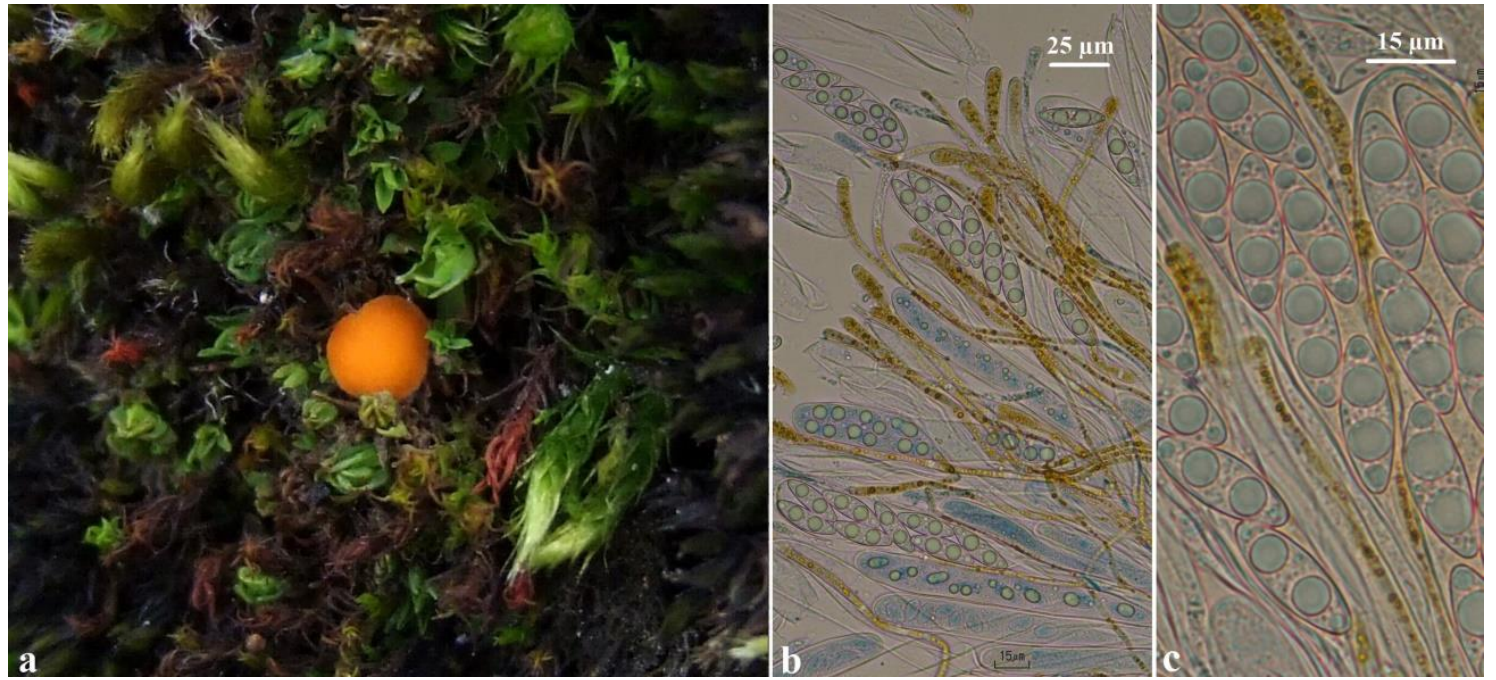

Figure 7. Octospora coccinea: a- ascocarp, b- asci and paraphyses, c- ascospores. 
3.8. Octospora excipulata (Clem.) Benkert, Mycologia Montenegrina 10: 10 (2008) (Figure 8)

Syn. Leucopezis excipulata Clem., Octospora roxheimii Dennis \& Itzerott, Octospora roxheimii var. aestivalis Caillet \& Moyne, Octospora roxheimii Dennis \& Itzerott, var. roxheimii.

Macroscopic and microscopic features: Apothecia 0.5-5 $\mathrm{mm}$ in diameter, pale orange to orange, lighter at the margin. Asci $150-220 \times 17-22 \mu \mathrm{m}$, cylindrical to clavate, eight spored, spores uniseriate. Paraphyses cylindrical, septate, enlarging towards the apex up to 5-7 $\mu \mathrm{m}$. Spores
17-27 $\times 12-17 \mu \mathrm{m}$, ellipsoid, smooth, hyaline, with a central large drop. Octospora excipulata grows in association with the moss Funaria hygrometrica Hedw. (Benkert, 2007; Eckstein and Eckstein, 2009).

Specimen examined: Nurdağı, Atmalı village, roadside, among Bryum dichotomum Hedw., $37^{\circ} 08^{\prime} \mathrm{N}-36^{\circ} 52^{\prime} \mathrm{E}, 620$ m, 04.04.2015, K.11619; Ataköy, roadside, among moss, $37^{\circ} 08^{\prime} \mathrm{N}-36^{\circ} 54^{\prime} \mathrm{E}, \quad 985 \mathrm{~m}, \quad 22.03 .2015, \quad \mathrm{~K} .11486$; Şehitkamil, Öğümsöğüt village, roadside, $37^{\circ} 07^{\prime} \mathrm{N}$ $37^{\circ} 19^{\prime} \mathrm{E}, 1110 \mathrm{~m}, 01.03 .2015$, K.11371; Gaziantep, Sahinbey, Gaziantep Zoo, among moss, $37^{\circ} 02^{\prime} \mathrm{N}-37^{\circ} 17^{\prime} \mathrm{E}$, 970 m, 15.11.2015, K.12788.
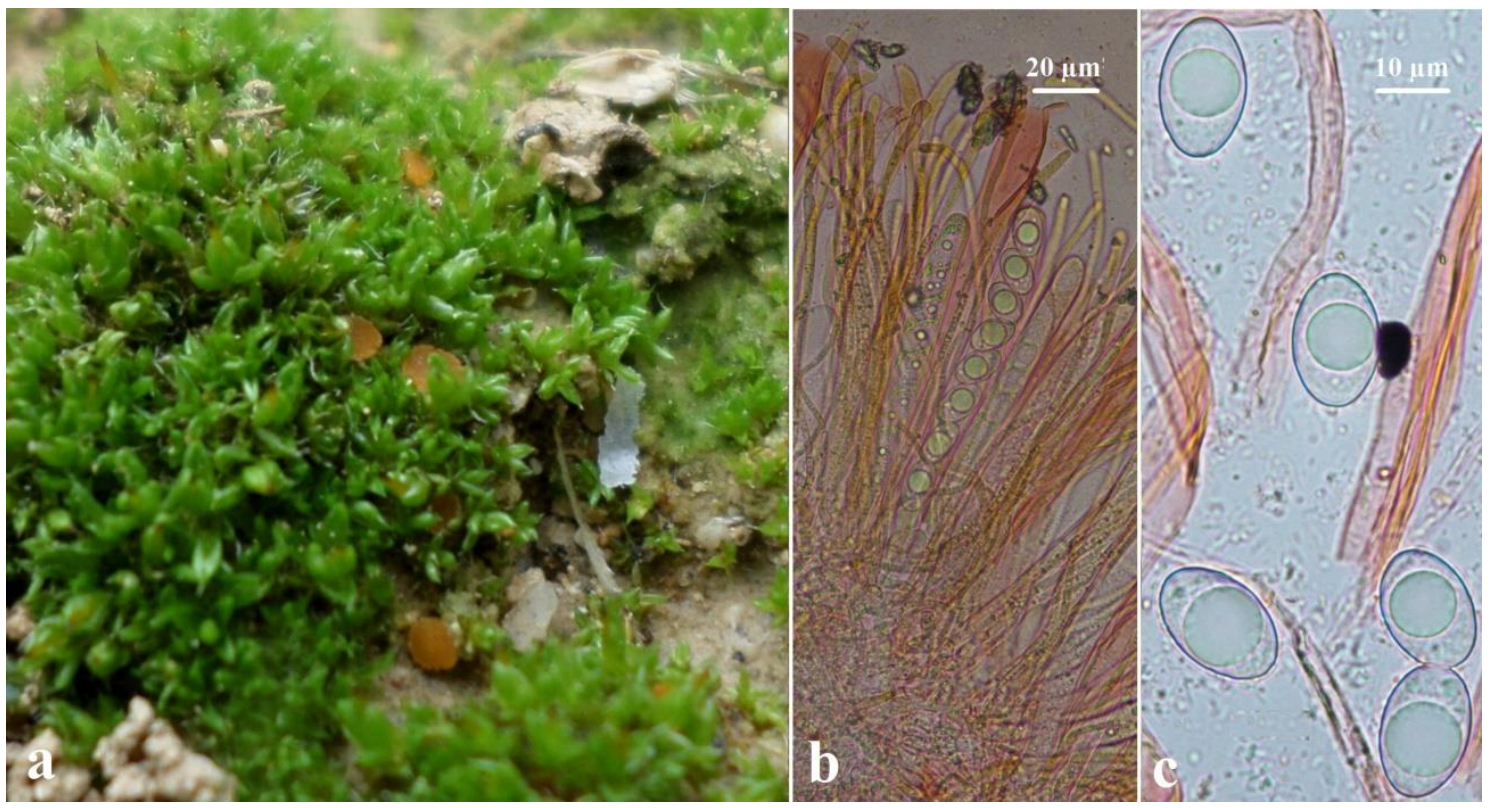

Figure 8. Octospora excipulata: a- ascocarps, b- asci and paraphyses, c- ascospores.

3.9. Octospora gemmicola Benkert, Öst. Z. Pilzk. 7: 49 (1998) (Figure 9)

Syn. Octospora gemmicola Benkert, var. gemmicola, Octospora gemmicola var. tetraspora Benkert.

Macroscopic and microscopic features: Apothecia 1-2.5 $\mathrm{mm}$ in diameter, cup to disc shaped with a weak membranaceous margin, hymenium plane to cupulate, orange. Asci $160-240 \times 16-23 \mu \mathrm{m}$, cylindrical to somewhat clavate, eight spored, spores mostly biseriate. Paraphyses cylindrical, enlarged toward the apex up to 5-8 $\mu \mathrm{m}$. Spores $18-25 \times 12-15 \mu \mathrm{m}$, narrowly ellipsoid to subfusiform, smooth, one to two (seldomly three) large and several small oil droplets. Octospora gemmicola was reported to grow among Bryum dichotomum Hedw., $B$. rubens Mitt., $B$. ruderale Crundw.\& Nyholm and $B$. radiculosum Brid. (Eckstein and Eckstein, 2009).

Specimen examined: Gaziantep, Şehitkamil, Dülükbaba picnic area, on Bryum sp, $37^{\circ} 07^{\prime} \mathrm{N}-37^{\circ} 19^{\prime} \mathrm{E}, 1110 \mathrm{~m}$, 01.03.2015, K.11367.

3.10. Octospora leucoloma Hedw., Descr. micr.-anal. musc. frond. (Lipsiae) 2: 13 (1789) (Figure 10)

Specimen examined: Gaziantep, Şahinbey, Yeşilkent cemetery, among Pterygoneurum ovatum (Hedw.) Dixon, $37^{\circ} 00^{\prime} \mathrm{N}-37^{\circ} 25^{\prime} \mathrm{E}, 865 \mathrm{~m}, 13.11 .2015$, K.12771; Nurdağ 1 ,
Kömürler village, roadside, $37^{\circ} 09^{\prime} \mathrm{N}-36^{\circ} 48^{\prime} \mathrm{E}, 535 \mathrm{~m}$, 03.04.2015, K.11593; Konya, city center, bus terminal, between the floor brick, on Bryum argenteum, $37^{\circ} 56^{\prime} \mathrm{N}$ $32^{\circ} 30^{\prime} \mathrm{E}, 1030 \mathrm{~m}, 04.12 .2017$, K.13944.

3.11. Octospora musci-muralis Graddon, Trans. Br. mycol. Soc. 58(1): 147 (1972) (Figure 11)

Syn. Octospora musci-muralis Graddon var. muscimuralis; Octospora musci-muralis var. neglecta (Dennis \& Itzerott) Benkert; Octospora neglecta Dennis \& Itzerott.

Macroscopic and microscopic features: Apothecia 1-4 $\mathrm{mm}$ in diameter, hymenium bright orange to orangebrown, margin paler and finely toothed. Asci 160-200 $\times$ 19-24 $\mu \mathrm{m}$, cylindrical to clavate, attenuated at the base, 8 spored, spores biseriate. Paraphyses filiform, apically curved and enlarged up to 4-5 $\mu \mathrm{m}$. Spores 20-25 × 9-10.5 $\mu \mathrm{m}$, elliptical to subcylindrical, hyaline, with two or one large drops. Octospora musci-muralis was reported to grow on the walls on the pads of Grimmia Hedw. sp. (Dennis and Itzerott, 1973; Eckstein and Eckstein, 2009) and Schistidium Brid. sp. (Benkert, 2007).

Specimen examined: Gaziantep, Şehitkâmil, cemetery, on Grimmia pulvinata (Hedw.) Sm., $37^{\circ} 04^{\prime} \mathrm{N}-37^{\circ} 23^{\prime} \mathrm{E}$, $845 \mathrm{~m}, 27.02 .2015$, K.11329; Cerityeniyapan village, $37^{\circ} 10^{\prime} \mathrm{N}-37^{\circ} 08^{\prime} \mathrm{E}, 1070 \mathrm{~m}, 07.03 .2015$, K.11395; Oğuzeli, cemetery, $36^{\circ} 57^{\prime} \mathrm{N}-37^{\circ} 30^{\prime} \mathrm{E}, 670 \mathrm{~m}, 08.03 .2015$, K.11428. 

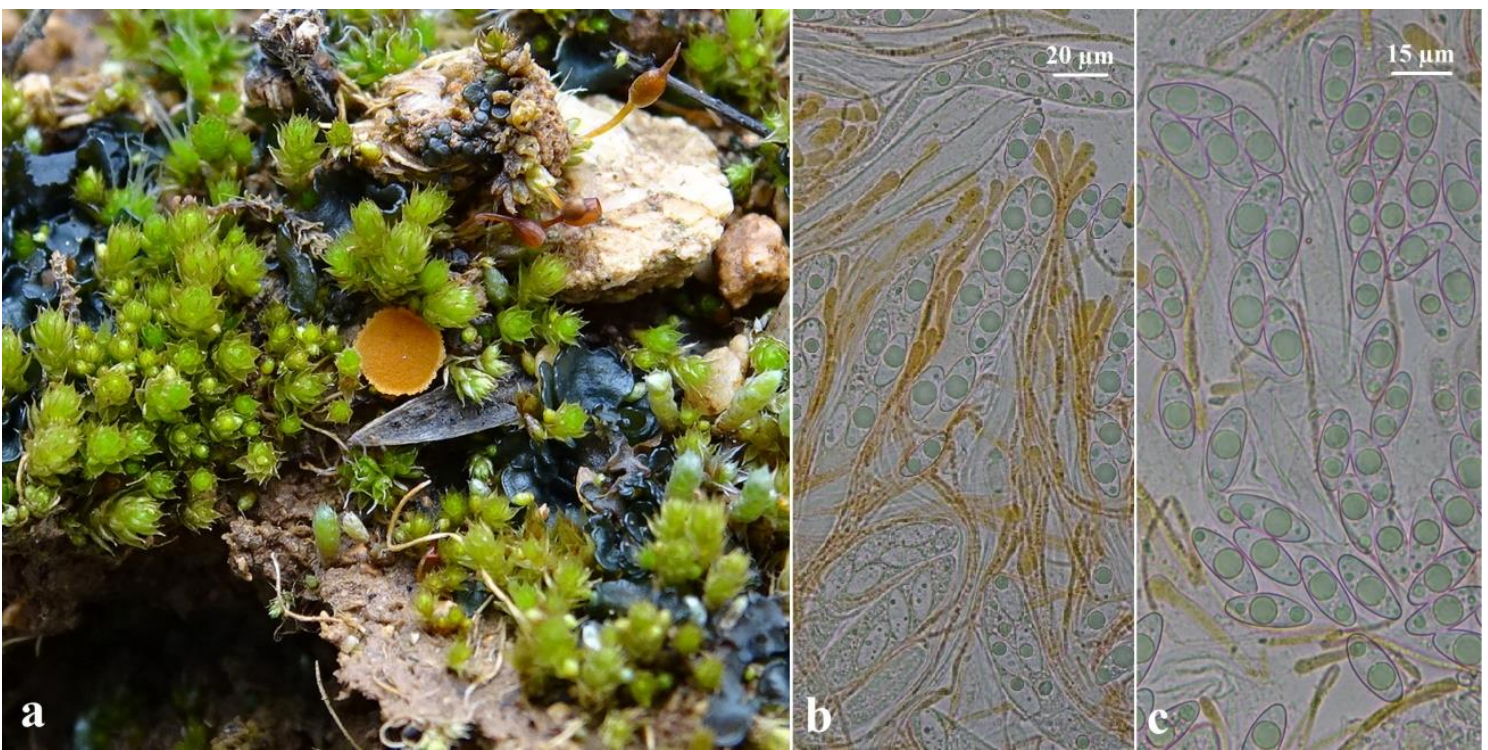

Figure 9. Octospora gemmicola: a- ascocarp, b- asci and paraphyses, c- ascospores.
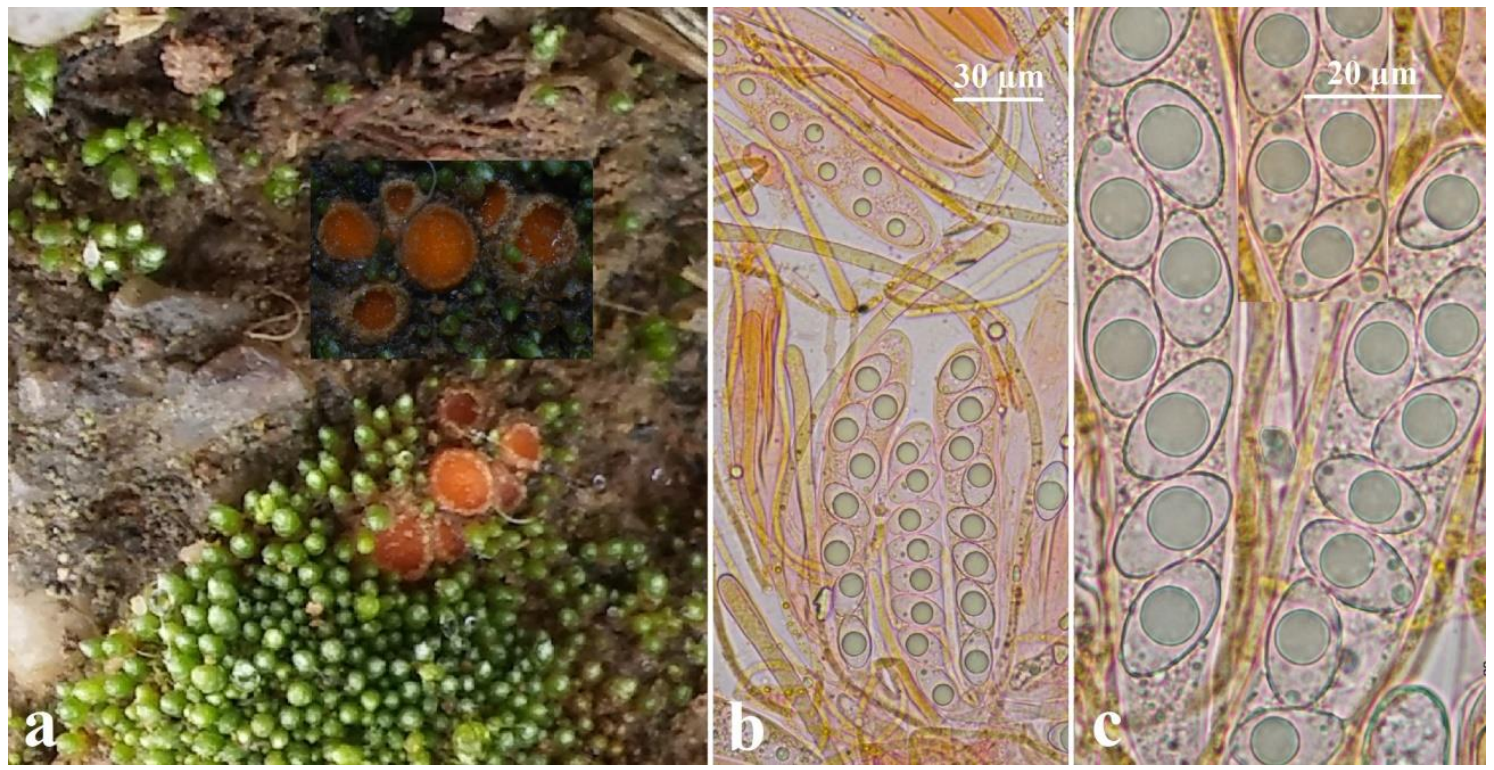

Figure 10. Octospora leucoloma: a- ascocarps, b- asci and paraphyses, c- ascospores.

3.12. Octospora orthotrichi (Cooke \& Ellis) K.B. Khare \& V.P. Tewari, Can. J. Bot. 56(17): 2118 (1978) (Figure 12)

Syn. Humaria orthotrichi (Cooke \& Ellis) Sacc., Humarina orthotrichi (Cooke \& Ellis) Seaver, Peziza orthotrichi Cooke \& Ellis.

Macroscopic and microscopic features: Apothecia 0.5-2 $\mathrm{mm}$ in diameter, cup to disc shaped, with inconspicuous membranaceous margin, hymenium pale orange to orange, margin paler. Asci 140-240 × 17-24 $\mu \mathrm{m}$, cylindrical, 8 spored, spores generally uniseriate. Paraphyses straight, septate, enlarged towards the apex. Spores 15-20 $\times 10-13$ $\mu \mathrm{m}$, ellipsoid to broadly ellipsoid, some with flattened one side, warted, with one or less often with two guttules. Though the exclusive association of Octospora orthotrichi with Orthotrichum diaphanum Brid. was reported (Benkert, 1998), it can also be found on the bark of trees or on stones, always between shoots of its host (Egertova et al., 2015).
Specimen examined: Gaziantep, Nurdağ 1 , Olucak village, on Orthotrichum diaphanum Schrad. ex Brid., $37^{\circ} 10^{\prime} \mathrm{N}$ $36^{\circ} 40^{\prime} \mathrm{E}, 950 \mathrm{~m}, 10.04 .2015$, K.11678.

3.13. Octospora polytrichi (Schumach.) Caillet \& Moyne, Bull. trimest. Soc. mycol. Fr. 96(2): 192 (1980) (Figure 13)

Syn. Barlaea polytrichi (Schumach.) Sacc.; Lamprospora polytrichi (Schumach.) Le Gal; Neottiella polytrichi (Schumach.) Massee; Peziza polytrichi Schumach.; Peziza turfosa Pers.; Sarcoscypha polytrichi (Schumach.) Höhn.

Macroscopic and microscopic features: Apothecia 1-3 $\mathrm{mm}$ in diameter, saucer to disc-shaped, resting stalkless on the ground. Hymenium orange to orange reddish. Outer surface lighter, margin smooth. Asci 230-250 × 19-21 $\mu \mathrm{m}$, cylindrical, tapering towards the base. Paraphyses cylindrical, forket at the base, septate. Spores 12-14 $\mu \mathrm{m}$ in diameter, round, hyaline and reticulately ornamented. Octospora polytrichi was reported to grow in association with Polytrichum Hedw. sp. (Wang and Kimbrough, 1992). 


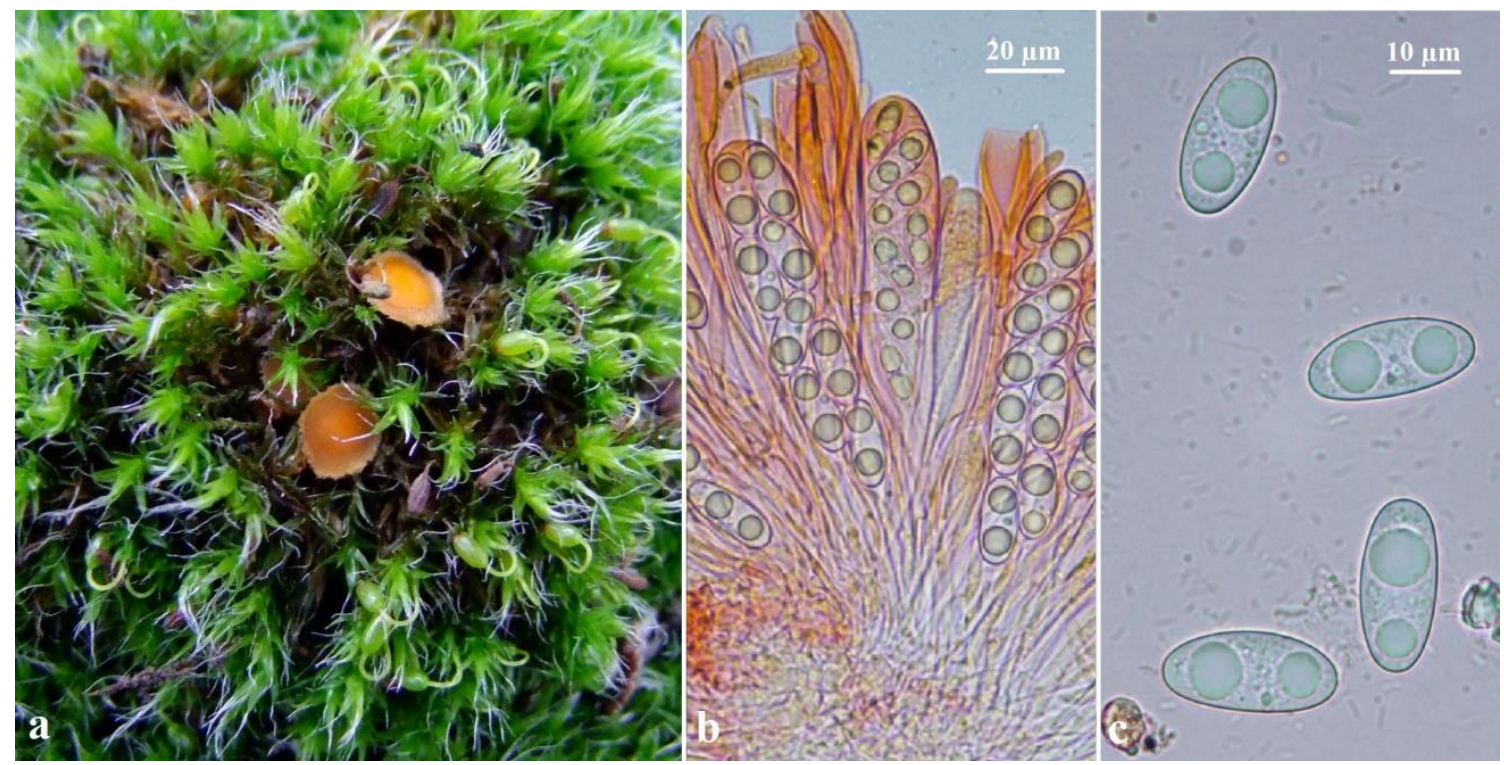

Figure 11. Octospora musci-muralis: a- ascocarps, b- asci and paraphyses, c- ascospores.
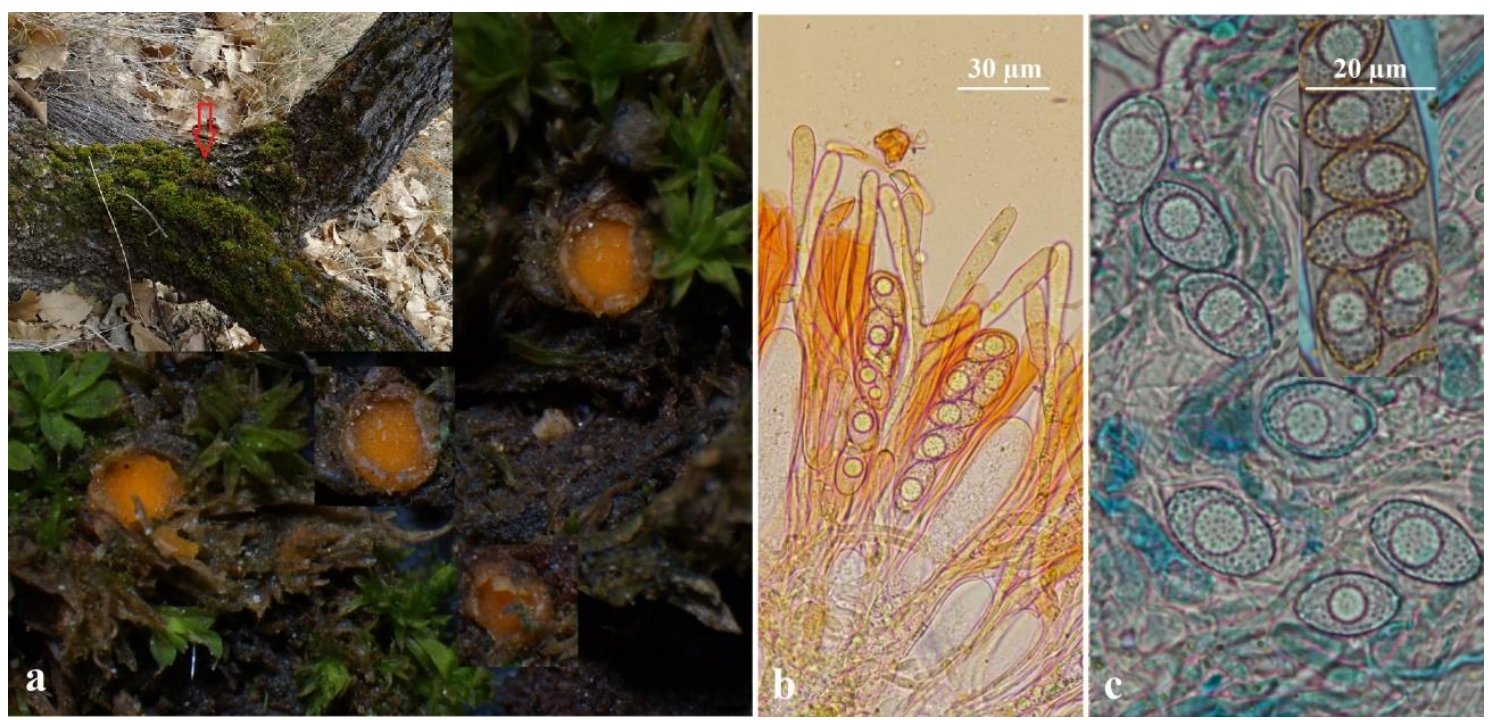

Figure 12. Octospora orthotrichi: a- ascocarps, b- asci and paraphyses, c- ascospores.
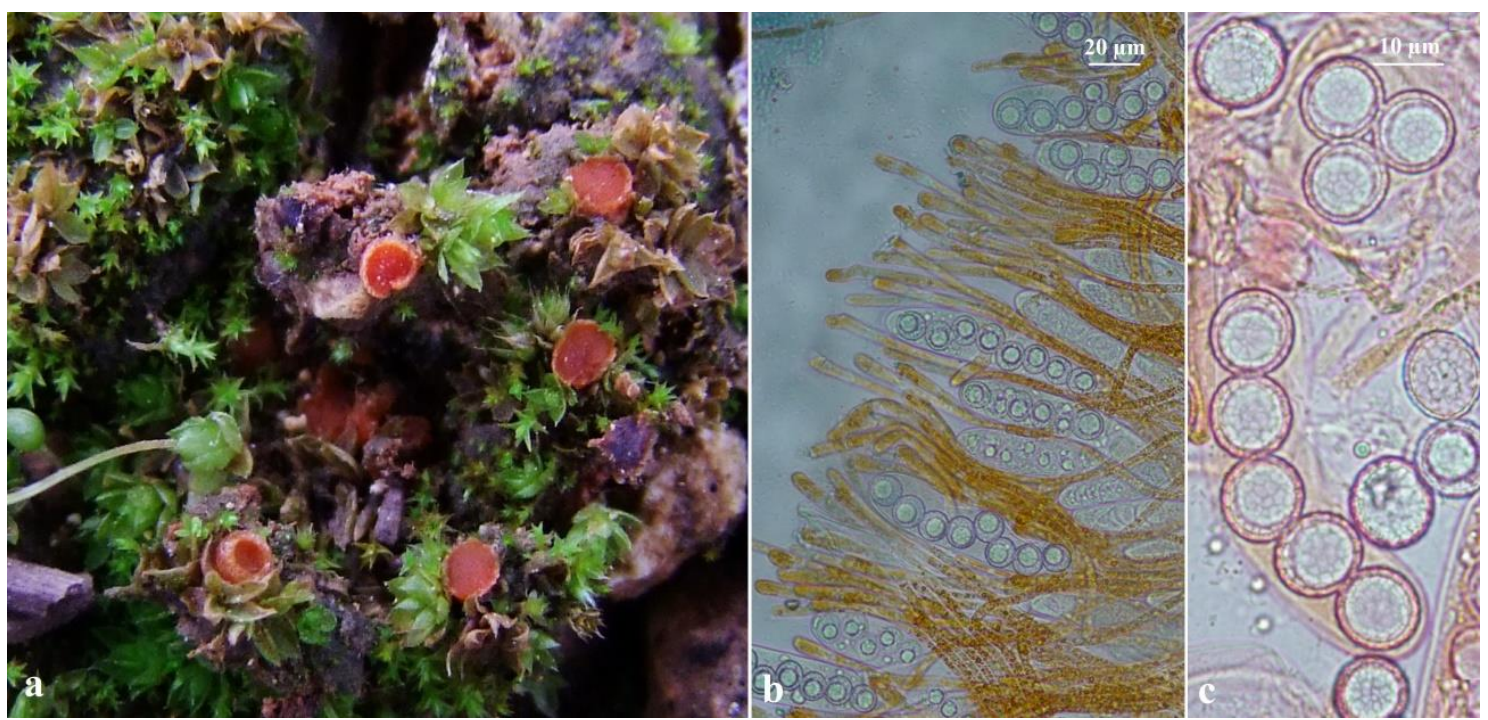

Figure 13. Octospora polytrichi: a- ascocarps, b- asci and paraphyses, c- ascospores. 
Specimen examined: Gaziantep, Nurdağı, Kömürler village, roadside, among Funaria hygrometrica Hedw., $37^{\circ} 09^{\prime} \mathrm{N}-36^{\circ} 48^{\prime} \mathrm{E}, 535 \mathrm{~m}, \mathrm{~K} .11581$.

3.14. Octospora rustica (Velen.) J. Moravec, Česká Mykol. 23(4): 226 (1969) (Figure 14)

Syn. Humaria rustica Velen.

Macroscopic and microscopic features: Apothecia 1-4 $\mathrm{mm}$ in diameter, at first cup shaped, soon becomes flat and disc shaped, hymenium orange, pale orange without a membranaceous margin. Asci 140-190 × 14-19 $\mu \mathrm{m}$, cylindrical, eight spored, spores uniseriate. Paraphyses cylindrical and enlarged up to 4-7 $\mu \mathrm{m}$ towards the apex. Spores 15-18 $\times 10-12 \mu \mathrm{m}$, ellipsoid, smooth, hyaline with a large drop. Octospora rustica grows on sandy soil on burnt ground among Ceratodon purpureus (Hedw.) Brid. (Dennis and Itzerott, 1973; Cailet and Moyne, 1989).

Specimen examined: Gaziantep, Nurdağı, Atmalı village, among Tortula acaulon (With.) R.H. Zander, $37^{\circ} 08^{\prime} \mathrm{N}$ $36^{\circ} 52^{\prime} \mathrm{E}, 620 \mathrm{~m}, 12.04 .2015$, K.11728.

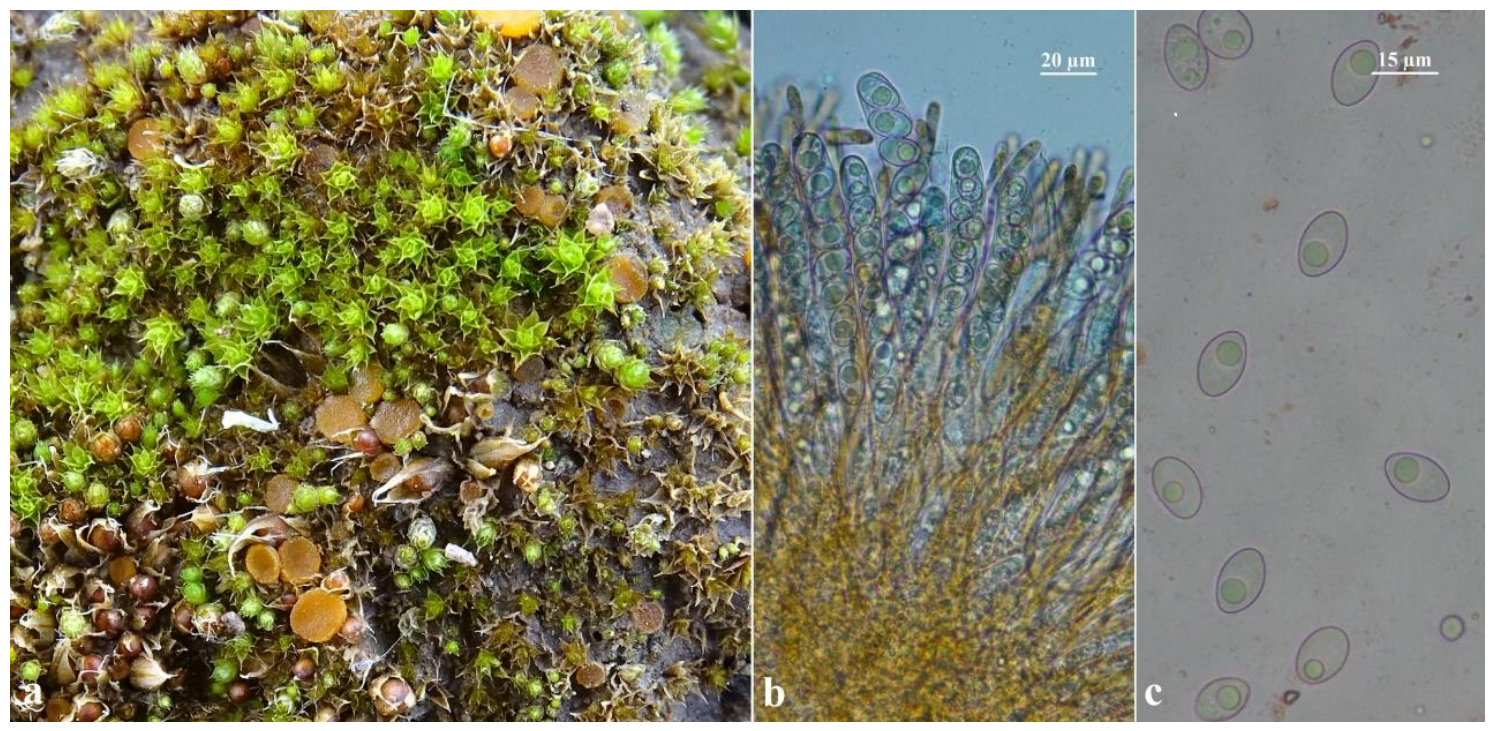

Figure 14. Octospora rustica: a- ascocarps, b- asci and paraphyses, c- ascospores.

\section{Discussion}

The habit is very similar in most bryophilous Pezizales with extremely small and more or less orange colored ascomata hidden between host plants or organs. That's why they are usually an easily overlooked group of fungi. Even for a keen and experienced observer it is a timeconsuming and troublesome task to detect them in field (Döbbeler, 1997). Due to the close similarities between macroscopic features, microscopic characteristics, especially the spores as being globose, subglobose, ellipsoid, fusiform, smooth or ornamented, are generally used as important diagnostic characters for the identification of them.

Though Lamprospora and Octospora have generally been differentiated from each other by globose and ellipsoid spores (Seaver, 1914; Yao and Spooner, 1996), here the systematics of Kirk et al. (2008) and the Index fungorum (www.indexfungorum.org; accessed 2 November 2017) were followed.

As a result of this study, two bryophilous ascomycete genera (Inermisia and Lamprospora) and thirteen bryophilous species within the order Pezizales (Inermisia 1, Lamprospora 3 and Octospora 9) were added as new records for the macromycota of Turkey, increasing the number of bryophilous ascomycete species of Turkey from 6 to 19. Among the determined species, Inermisia gyalectoides resembles Sepultaria semi-immersa (P. Karst.) Massee, in terms of habitat and morphology. But it differs with colorless and thin-walled anchoring hyphae, and less fusoid and shorter ascospores. It also recalls Inermisia pilifera (Cooke) Dennis \& Itzerott, from which it differs with larger and broder ascospores (Dennis and Itzerott, 1973).

Due to the spore similarity, Lamprospora carbonicola has generally been misinterpreted and frequently confused with $L$. dictydiola. However, the habitat and the host of latter species makes it easy to distinguish each other (Benkert, 1987). This species also has morphological similarities with Pulvinula mussooriensis (K.S. Thind, E.K. Cash \& Pr. Singh) L.R. Batra. But P. mussoriensis differs with yellow apothecia and smaller spores (Pradhan et al., 2013; Eckstein and Eckstein, 2013).

Seaver (1914) has noted the similarity of Octospora areolata to the Moravecia calospora (Quél.) Benkert, Caillet \& Moyne, but round and non-elliptic spores of $O$. areolata distinguishes it from the latter species.

Octospora musci-muralis may grow in association with cushions of Grimmia sp., where O. grimmia Dennis \& Itzerott generally exists. However $O$. music-muralis is easily recognizable by its elliptic cylindric, smooth and biguttulate ascospores (Dennis and Itzerott, 1973).

Octospora orthotrichi has a special habitat, Orthotrichum sp., but $O$. affinis Benkert \& L.G. Krieglst may also parasitize the mosses within the same genus. However, smaller, ellipsoid to subglobose spores and exclusive parasiticity of $O$. affinis on Orthotrichum affine Schrad. ex Brid. differs it from O. orthotrichi (Egertova et al., 2015).

Morphological and ecological characteristics of the determined species generally agree with those given in literature. But some ecological differences were also determined. For example, O. areolata were reported to 
grow on some mosses belonging to the genera Bryum and Pohlia, but our sample was determined on Syntrichia ruralis. Here we determined the substrate of Octospora exipulata as Bryum dichotomum, while it was reported on Funaria hygrometrica. Likewise, Ptychostomum donianum, Pterygoneurum ovatum and Tortula acaulon were also determined as new hosts for $O$. axillaris and $O$. rustica respectively.

\section{Acknowledgements}

The authors would like to thank TÜBİTAK for supporting the project (212T112) financially, and Prof. Dr. Güray Uyar for identifying the bryophyte samples.

\section{References}

Akata I, Kaya A (2013). Three pyronemataceous macrofungi genera new to Turkish Mycota. Turkish Journal of Botany 37(5): 977-980.

Benkert D (1987). Contributions to the taxonomy of the genus Lamprospora (Pezizales). Zeitschrift für Mykologie 53(2): 195272.

Benkert D (1997). New Species of the Genus Octospora. Beiträge zur Kenntnis der Pilze Mitteleuropas XI: $35-42$.

Benkert D (1998). Beiträge zur Kenntnis bryophiler Pezizales-Arten. 8. Viersporige Taxa der Gattung Octospora. Österreichische Zeitschrift für Pilzkunde 7: 39-63.

Benkert D (2007). Knowledge of the Occurrence of Bryophilous Pezizales (Ascomycotina) in South-East Europe. Mycologia Montenegrina 10: 7-21.

Benkert D, Brouwer E (2004). New Species of Octospora and Some Further Remarkable Bryoparasitic Pezizales From the Netherlands. Persoonia 18(3): 381-391.

Beug MW, Bessette AE, Bessette AR (2014). Ascomycete Fungi of North America. A Mushroom Reference Guide.

Breitenbach J, Kränzlin F (1984). Fungi of Switzerland Volume 1. Lucerne: Verlag Mykologia.

Caillet M, Moyne G (1988-89). Bull. Soc. Hist. nat Doubs 84: 9-24.

Cannon PF, Kirk PM (2007). Fungal Families of the World. Wallingford, UK: CAB International.

Çolak ÖF, Kaygusuz O (2017). Octospora leucoloma (Pyronemataceae): a new bryoparasitic genus record for Turkish mycobiota. Phytologia Balcanica 23(3): 345-348.

Dennis RWG, Itzerott H (1973). Octospora and Inermisia in Western Europe. Kew Bulletin 28: 5-23.

Döbbeler P (1997). Biodiversity of bryophilous ascomycetes. Biodiversity and Conservation 6: $721-738$.

Dougoud R, Roth JJ (1972). Octospora musci-muralis Graddon. Trans. Brit. Mycol. Soc. 58: 97-103.

Eckstein J (2014). Lamprospora hispanica and Lamprospora tuberculatella - two bryoparasitic discomycetes (Ascomycota, Pezizales) new to Germany. Zeitschrift für Mykologie 80(1): 105-117.

Eckstein J, Eckstein G (2009). Bryoparasitic Pezizales (Ascomycetes) of the genera Lamprospora, Octospora and Neottiella in the Old Botanical Garden at Göttingen (Germany, Lower Saxony). Herzogia 22: 213-228.

Eckstein J, Eckstein G (2013). Noteworthy findings of bryoparasitic Pezizales (Ascomycota) from Germany. Boletus 34: 55-66.

Eckstein J, Eckstein G, Vega, M (2014). Bemerkenswerte Funde bryoparasitischer Pezizales (Ascomycota) aus Deutschland II. Boletus 35: 17-25.

Egertova Z, Eckstein J, Vega M (2015). Lamprospora tuberculata, Octospora ithacaensis, O. orthotrichi and O. affinis - four bryoparasitic ascomycetes new to the Czech Republic. Czech Mycology 67: 119-133.

Felix H (1988). Fungi on bryophytes, a review. Botanica Helvetica 98: 239-269.

Fox HF, Grant PJ, Moore CR (1994). Octospora musci-muralis Graddon, (Ascomycotina: Humariaceae) in Ireland. The Irish Naturalists Journal 24(9): 378-379.

Hansen K, Pfister DH (2006). Systematics of the Pezizomycetes: The Operculate Discomycetes. Mycologia 98(6): 1029-1040.

Hughes JG (1982). Penetration by rhizoids of the moss Tortula muralis Hedw. into well cemented oolitic limestone. International Biodeterioration Bulletin 18: 43-46.

Index Fungorum (2017). http://www.indexfungorum.org/Names/Names.asp. Accessed 2 November 2017.

Itzerott H (1977). The genus Octospora and an attempt to solve its taxonomic problems. Kew Bulletin 31: 497-500.

Kaya A, Uzun Y, Karacan İH, Yakar S (2016). Contributions to Turkish Pyronemataceae from Gaziantep province. Turkish Journal of Botany 40(3): 298-307.

Khare KB (1976). Some Lamprospora species from India. Norwegian Journal of Botany 23: 1-5.

Khare KB (2003). Descriptions of and comments on some species of Octospora and Kotlabaea (Pezizales, Humariaceae). Nova Hedwigia 77: 445-485.

Khare KB and Tewari VP (1975). The Genus Octospora and Its Typification. Mycologia 67(5): $972-979$.

Kirk PM, Cannon PF, Minter DW, Stalpers JA (2008). Dictionary of the Fungi. 10th ed. Wallingford, UK: CAB International.

Le Gal M (1940). Lamprospora polytrichi (Fr. ex Schum.). Bulletin trimestriel de la Société mycologique de France 56: 39-45.

Medardi G (2006). Atlante fotografico degli Ascomiceti d'Italia. A.M.B. Fondazione, Centro Studi Micologici, Italia. 
Pradhan P, Dutta AK, Acharya K (2013). Pezizales of West Bengal, India I. Pyronemataceae: Lamprospora and Miladina. Asian Journal of Microbiological Biotechnology Environmenatl Science 15: 491-494.

Schumacher TK (1993). Studies in arctic and alpine Lamprospora species. Sydowia 45(2): 307-337.

Seaver FJ (1912). The Genus Lamprospora, with Descriptions of Two Species. Mycologia 4: 45-48.

Seaver FJ (1914). A Preliminary Study of the Genus Lamprospora. Mycologia 6: 5-24.

Seaver FJ (1942). The North American Cup-Fungi (Operculates). Supplementary Edition. New York, NY, USA.

Senn-Irlet B (1988). Zum Nachweis der Bryoparasitischen Lebensweise von Octospora orthotricha und O. tetraspora (Pezizales, Ascomycetes). Mycologia Helvetica 3: 173-181.

Sesli E, Denchev CM (2014). Checklists of the Myxomycetes, Larger Ascomycetes, and Larger Basidiomycetes in Turkey. 6th edn. Mycotaxon Checklists Online. (http://www.mycotaxon.com/resources/checklists/sesli-v106-checklist.pdf): 1-136.

Solak MH, Işıloğlu M, Kalmış E, Allı H (2015). Macrofungi of Turkey, Checklist, Vol. II. İzmir, Turkey: Üniversiteliler Ofset.

Stephenson SL, Studlar SM (1985). Myxomycetes fruiting upon bryophytes: coincidence or preference? Journal of Bryology 13(4):537-548.

Svrček M, Kubička J (1961). Some operculate Discomycetes from the vicinity of the pond 'Dvoriste' in southern Bohemia. Ceská Mykologie. 15(2):61-77.

Türkoğlu A, Yağız D (2012). Contributions to the macrofungal diversity of Uşak Province. Turkish Journal of Botany 36(5): 580589.

Uzun Y, Karacan İH, Yakar S, Kaya A (2017). Octospora Hedw., A new genus record for Turkish Pyronemataceae. Anatolian Journal of Botany 1(1): 18-20.

Wang YZ, Kimbrough JW (1992). Monographic studies of North American species of Octospora previously ascribed to Lamprospora (Pezizales, Ascomycetes). Special Publication no. 4, National Museum of Natural Science. Taiwan.

Wieschollek D (2013). Octospora gemmicola var. tetraspora Benkert, a bryoparasitic discomycete with four-spored asci new for Saxony-Anhalt. Boletus 34: 67-71.

Yao YJ, Spooner BM (1996). Notes on British species of Octospora. Mycologiacal Research 100: 175-178.

Yılmaz F, Öder N, Işıloğlu M (1997). The macrofungi of the Soma (Manisa) and Savaştepe (Balıkesir) districts. Turkish Journal of Botany 21(4): 221-230.

Cite this article: Uzun Y, Karacan İH, Yakar S, Kaya A (2018). New bryophillic Pyronemataceae records for Turkish Pezizales from Gaziantep province. Anatolian Journal of Botany 2(1): 28-38. 